\title{
Propuesta para el Análisis de Diseños Aumentados en Fitomejoramiento: Un Caso en Papa
}

\author{
José M. Cotes T. ${ }^{1}$, Carlos E. Ñústez L. ${ }^{2}$
}

\section{Resumen}

El uso de diseños aumentados en las etapas tempranas, en los programas de fitomejoramiento, son una herramienta valiosa para las decisiones de selección, pero en general es poco lo que se implementa en estos programas. Cuando se utilizan, es común seleccionar los genotipos que se evalúan, como iguales o superiores al mejor, al peor o al promedio de los testigos utilizados. En este trabajo se propone un método de análisis, utilizando la suma de rangos propuesta por Kang (1991), desarrollando así un análisis de diseños aumentados, bajo un diseño en bloques completos al azar, el cual permite realizar análisis de una o varias variables, siendo esta última su ventaja más importante.

En todas las variables evaluadas se observó cómo el método propuesto realizó una mejor selección de genotipos, reduciendo su número, con respecto al método tradicional. Finalmente se desarrolló una MAGRO, en el programa SAS, que permite analizar diseños aumentados por ambos métodos.

\section{Palabras claves adicionales: Solanum tuberosum, fitomejoramiento, diseños experimentales, selección.}

\section{Proposal for the Augmented Design Analysis in Plant Breeding: A Potato Case}

\section{Summary}

The use of augmented designs in phases of plant breeding programs is a valuable tool for the selection decisions, but as a rule very little of

1 Candidato a M.Sc. Facultad de Agronomía. Universidad Nacional de Colombia. Santafé de Bogotá D.C. Colombia, jmcotes@unal.edu.co.

2 Profesor Asociado. Universidad Nacional de Colombia. Santafé de Bogotá D.C. Colombia. cenuztezl@unal.edu.co 
these methods are implemented in those programs. When they are used, it is common to select the genotypes that are evaluated, as equal or superior to the best, to the worst, or to the average of the controls utilized. In this study, an analysis using the method proposed by Kang (1991) was made, thus developing an augmented design analysis, under a random complete blocks design, which permits to analyze one or more variables, being the latter its more important advantage.

In all the evaluated variables it was observed how the proposed method accomplished a better selection of genotypes, reducing its number with respect to the traditional method. Finally, a MAGRO that was developed for the SAS program, allowed the analysis of augmented designs by both methods.

Additional index words: Solanum tuberosum, plant breeding, experimental designs, selection.

\section{Introducción}

En estados tempranos de selección, en los programas de fitomejoramiento, es usual realizar selección visual de los genotipos bajo el criterio del investigador, dando prioridad a los caracteres agronómicos objeto del programa. Este tipo de selección se hace por diferentes circunstancias, tales como: insuficiente cantidad de semilla para poder realizar un diseño experimental convencional, alto número de líneas o genotipos a evaluar, con lo cual, adicionalmente el área requerida para ello, se convierte en limitante. Al no poderse realizar un análisis de varianza, la eliminación de algunas líneas por rendimiento está influenciada por la posición que le correspondió en el lote.

Por las anteriores consideraciones y en razón al incremento en los costos de investigación, se hace necesario que los mejoradores estén buscando constantemente formas para perfeccionar la eficiencia de un programa de investigación (Pérez y Santamaría, 1990).

Los diseños aumentados han sido desarrollados básicamente por Federer (1956), quién menciona que uno de los problemas principales en fitomejoramiento, fitopatología, etc., es la evaluación 
de los nuevos genotipos, herbicidas, pesticidas, etc., donde se requiere el uso de diseños eficientes para evaluar los tratamientos, cuando por razones de costo o porque el número de tratamientos es tan alto que sólo se puede repetir una vez. Situaciones experimentales como estas, se presentan en la introducción de un material vegetal, donde la cantidad de semilla es mínima y solamente permite una repetición o cuando las actividades de un programa de mejoramiento incluyen el desarrollo y tamizado de un alto número de líneas, las cuales se siembran en uno o pocos surcos y necesitan ser evaluadas entre si o comparadas con los testigos. En estos casos Federer (1961) señala que el diseño experimental seleccionado debe ajustarse a las condiciones experimentales, en lugar de que los tratamientos (genotipos) y las unidades experimentales se ajusten a los requerimientos del diseño experimental (Pérez y Santamaría, 1990).

Cuando algunos testigos son repetidos " $r$ " veces $y$, las nuevas variedades son repetidas menos veces que aquellos, se pueden usar los diseños aumentados (Federer y Raghavarao, 1975). Estos diseños presentan mínima variación para la estimación del efecto de contrastes de testigos, de las variedades nuevas, de nuevas versus testigos y de los testigos y nuevas simultáneamente (Pérez y Santamaría, 1990).

Comúnmente en el análisis de diseños incrementados se compara el genotipo nuevo con la mejor, la peor o el promedio de las variedades testigo, dependiendo de la presión de selección y de los objetivos del programa de mejoramiento.

Un adecuado balance de las tasas de error tipo I y tipo II es de vital importancia en las decisiones de selección (Kang, 1996). Se ha encontrado que utilizando un nivel de significancia $(\alpha)$ de 0.10 se obtiene la mejor relación entre error tipo I y II (Glaz y Dean, 1988; Kang, 1993; Kang y Pham, 1991; Kang y Magari, 1996). En Ios diseños aumentados aplicados al mejoramiento vegetal, la hipótesis nula es Ho: $\mu_{a j-n}=\mu_{t}$, donde $\mu_{a j-n}$ es la media del genotipo nuevo ajustada y $\mu_{t}$, es la media de la variedad testigo de interés (mejor, peor o promedio). Si reducimos $\alpha$, cometemos mayor cantidad de error tipo II, es decir, seleccionamos genotipos como superiores al testigo, cuando en realidad no lo son. Si aumentamos el $\alpha$, se incrementa el error tipo I, donde concluimos que un genotipo es igual al testigo cuando en realidad es superior o inferior. Así, el cometer error tipo II es más perjudicial para las decisiones de selección, en estados iniciales de mejoramiento, que el incurrir en error tipo I. 
Una limitante del uso de diseños aumentados en estados tempranos de selección, es que, en general sólo se evalúa rendimiento total o comercial del nuevo genotipo. Si se está interesado en otras variables como altura, resistencia a enfermedades, etc., no se ha establecido una técnica de análisis multivariado para ello. Así, sólo se puede realizar selección por una variable. El uso de metodologías no paramétricas para el análisis multivariado han tenido escaso desarrollo y utilización (Puri y Sen, 1971). Kang (1991) aplicó el uso de la suma de rangos en la formación de un índice de selección por rendimiento y estabilidad en el análisis de la interacción genotipo por ambiente. Este es el punto de partida del presente artículo el cual propone implementar una metodología, utilizando esta técnica de suma de rangos, para el caso del uso de diseños aumentados, usando como modelo un caso en papa.

\section{Metodología}

El diseño más común en experimentación agrícola es el de bloques completos al azar (DBCA), bajo el cual se puede realizar un diseño incrementado (Federer, 1961). Así para un diseño incrementado bajo un DBCA se realizan los siguientes cálculos:

1. Se obtiene el ANOVA para las variedades testigo.

2. Se corrige el valor observado de cada genotipo nuevo así:

$Y_{i j}{ }^{\prime}=Y_{i j}-F C$ donde; $Y_{i j}$ ' es el valor ajustado de la variable evaluada para el j-ésimo bloque del i-ésimo genotipo, $Y_{i j}$ es el valor observado de la variable evaluada y FC es el factor de corrección calculado para cada bloque, con el cual se corrigen todos los genotipos de su respectivo bloque.

$$
F C=(l / g) *\left(\sum_{i, j} Y i j-\sum_{i, j} Y \mathrm{ij} / r\right) \text { Donde "g" es el }
$$

número de variedades testigo y "r" es el número de bloques.

Hay que tener en cuenta que, los grados de libertad del error para el ANOVA de las variedades testigo, debe ser mayor de 10, por lo cual, debe cumplirse la siguiente relación: $r>[10 /(c-1)+1]$, siendo "c" el número de testigos.

La metodología de análisis propuesta consta de los siguientes pasos: 
1. Realizar el análisis convencional para el diseño incrementado, pasos 1 y 2 anteriormente mencionados.

2. Ordenar los genotipos nuevos ajustados, de mayor a menor. Se ranquea el genotipo de menor valor con 1 , el segundo inferior con 2 y así sucesivamente, obteniendo la variable $\mathrm{R}$.

3. Elegir el testigo a probar (el mejor, el peor o el promedio) y, calcular la diferencia $(F)$ de este con todos los genotipos nuevos ajustados.

4. Obtener la diferencia mínima significativa (DMS) ajustada mediante la expresión:

$$
\mathrm{DMS}=\mathrm{t}_{\mathrm{a} / 2 . \mathrm{gle}}{ }^{*} \sqrt{\left((r-1) *(g-1) * C M E /\left(r^{*} g\right)\right)}
$$

donde "t" es el valor de Student, "gle" son los grados de libertad del error para el ANOVA de las variedades testigo y CME es el cuadrado medio del error para el mismo ANOVA.

5. Obtener $R$ ajustado ( $\left.R^{\prime}\right)$ de acuerdo a la DMS, asignando \pm 1 , cuando $F$ del genotipo ajustado esté entre $0 \mathrm{y} \pm 1 \mathrm{DMS}$, asignando \pm 2 , cuando $F$ del genotipo ajustado esté entre \pm 1 y +2 DMS, y así sucesivamente.

6. Obtener el IS (índice de selección), para lo cual se suman el rango ajustado ( $\left.R^{\prime}\right)$ y el rango natural $(R)$ de cada genotipo.

7. Calcular el promedio del IS y seleccionar los genotipos cuyo valor sea superior a éste.

Cuando se ha evaluado más de una variable, el procedimiento a seguir es:

- Realizar los pasos 1 a 7 anteriores, lo cual permite hacer inferencias por variable individual.

- Sumar los IS de cada variable y obtener el estadístico ISG (índice de selección general).

- Calcular el promedio del ISG y seleccionar los genotipos cuyo valor sea superior a éste.

Como vemos, el método se convierte en una combinación de coeficientes lineales de las variables evaluadas. Además, permite 
ponderar la importancia de cada variable de la siguiente manera. Supongamos que el investigador evalúa rendimiento, altura de planta y tamaño de grano y, la variable de mayor importancia es rendimiento. Las restantes se consideran con la misma importancia. Sí duplicamos el valor de IS para rendimiento y mantenemos el de las otras variables, obtenemos una proporción de 2:1:1, es decir, $50 \%$ de la decisión está influenciada por el rendimiento y el $25 \%$ por cada una de las otras dos variables. Esta flexibilidad del método, ofrece una herramienta valiosa para que el investigador decida ponderar a voluntad las variables evaluadas $\mathrm{y}$, por lo tanto, complementar importantemente la decisión de selección, según su criterio o necesidad.

\section{Programa SAS para el análisis de datos}

Para el procesamiento de la información se desarrolló una MAGRO en el programa SAS versión 6.12, lo cual facilita su uso y aplicación. El nombre de la macro es DA (Diseño Aumentado) y requiere cuatro parámetros para su utilización:

\% DA (DATA=, RESPONSE=, DECISION=, ALPHA=);

1. DATA: Es el archivo SAS donde están los datos, éste debe contener una variable llamada "CAT" en la cual se encuentran clasificados los genotipos como "nuevos" o "testigos", una variable 'TRAT' donde se nombran los genotipos (sin limite de caracteres) y una variable 'BLOQUE', en la cual se indica el número de bloque dentro de un diseño de bloques completos al azar.

2. RESPONSE: El nombre de la variable de respuesta a analizar (Máximo de 4 letras).

3. DECISION: Indica si la comparación se hace contra el mejor, el peor o el promedio de los testigos. Así éste tiene tres opciones MAX, MIN y MEAN.

4. ALPHA: Define el alfa para las pruebas de hipótesis. 
La macro arroja los siguientes resultados:

1. Análisis de Varianza para las variedades testigo incluyendo la prueba de comparación múltiple por DMS.

2. La media de la variable de respuesta ajustada para los genotipos nuevos, la DMS ajustada para comparar el nuevo genotipo contra el mejor, el peor o el promedio de los genotipos testigos y, el índice de selección siguiendo la metodología de suma de rangos propuesta por Kang (1991).

3. Una tabla de distribución de frecuencias de dos vías comparando selección tradicional con la selección realizada por el método propuesto.

La MACRO DA, además, genera un archivo SAS cuyo nombre comienza con IS'xxx' y termina con el nombre de la variable respuesta a evaluar. P.e. Si la variable respuesta se llama 'PT el archivo SAS se llamará ISPT. En este archivo SAS se encuentran todos los valores para calcular el índice de selección IS. En el se generan las variables AJ'xxx', TRAD'xxx', RDMS'xxx', RANK'xxx', IS'xxx' y SELC'xxx' que corresponden respectivamente a las variables respuesta ajustada para los genotipos nuevos, la decisión tomada por el método tradicional (INFERIOR, IGUAL o SUPERIOR), al ranqueo con base en la DMS ajustada, al ranqueo de los rendimientos, al índice de selección, y la decisión de selección tomada con el método propuesto (SI o NO). La MACRO funciona para el análisis de cualquier número de genotipos nuevos y cualquier número de testigos, si se cumple el requisito de gle $\geq 10$. 
El programa SAS, es el siguiente:

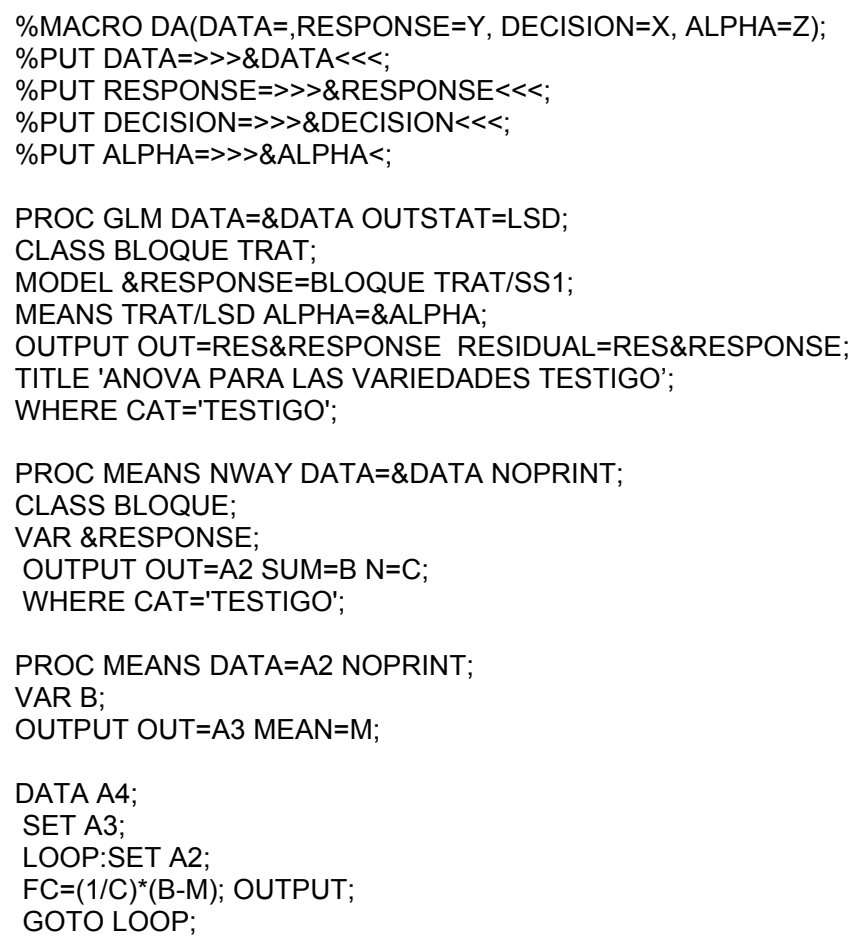

PROC SORT DATA=A4; BY BLOQUE;

DATA A5;

SET \&DATA;

IF CAT='TESTIGO' THEN DELETE;

PROC SORT DATA=A5; BY BLOQUE;

DATA A6;

MERGE A5 A4; BY BLOQUE;

$A J \& R E S P O N S E=\& R E S P O N S E-F C$;

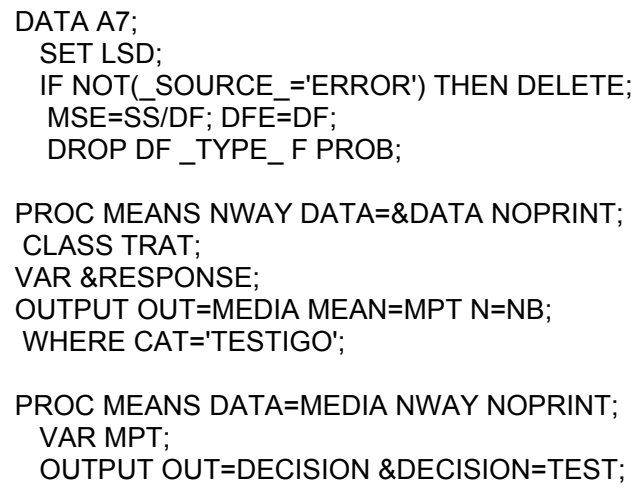




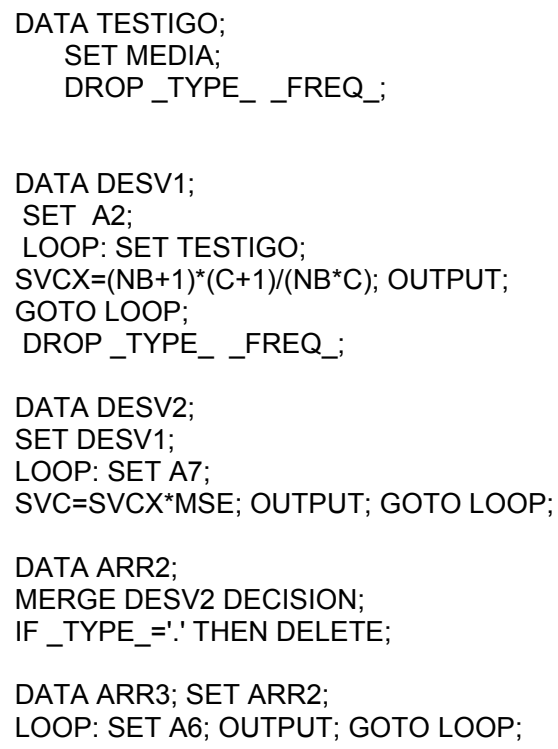

DATA PRUEBAST; SET ARR3;

DIF=AJ\&RESPONSE-TEST; T=DIF/(SVC**0.5); PROBT=(1-PROBT(ABS(T), DFE) ${ }^{\star} 2$; $\mathrm{T}=\mathrm{TINV}((1-(\& A L P H A / 2)), \mathrm{DFE}) ; \mathrm{LSD}=\mathrm{T}^{*}(\mathrm{SQRT}(\mathrm{SVC}))$;

IF 20*LSD $<A B S$ (DIF) $<=21 *$ LSD THEN RDMS\&RESPONSE $=21$; IF $19^{*}$ LSD $<A B S(D I F)<=20 *$ LSD THEN RDMS\&RESPONSE $=20$; IF $18^{*} \mathrm{LSD}<\mathrm{ABS}$ (DIF) $<=19^{*} \mathrm{LSD}$ THEN RDMS\&RESPONSE $=19$; IF $17^{*} \mathrm{LSD}<\mathrm{ABS}$ (DIF)<=18*LSD THEN RDMS\&RESPONSE $=18$; IF $16^{*}$ LSD $<A B S(D I F)<=17^{*}$ LSD THEN RDMS\&RESPONSE $=17$; IF $15^{*} \mathrm{LSD}<\mathrm{ABS}$ (DIF) $<=16^{*} \mathrm{LSD}$ THEN RDMS\&RESPONSE $=16$; IF $14^{*} \mathrm{LSD}<A B S$ (DIF) $<=15^{*} \mathrm{LSD}$ THEN RDMS\&RESPONSE $=15$; IF $13^{*}$ LSD $<A B S(D I F)<=14^{*}$ LSD THEN RDMS\&RESPONSE $=14$; IF $12^{*}$ LSD $<A B S(D I F)<=13^{*}$ LSD THEN RDMS\&RESPONSE $=13$; IF $11^{*} \mathrm{LSD}<\mathrm{ABS}$ (DIF) $<=12^{*} \mathrm{LSD}$ THEN RDMS\&RESPONSE $=12$; IF $10^{*} \mathrm{LSD}<\mathrm{ABS}$ (DIF) $<=11^{*} \mathrm{LSD}$ THEN RDMS\&RESPONSE $=11$; IF 9*LSD $<A B S(D I F)<=10^{*}$ LSD THEN RDMS\&RESPONSE $=10$; IF $8 *$ LSD $<A B S(D I F)<=9 *$ LSD THEN RDMS\&RESPONSE $=9$; IF $7 *$ LSD $<A B S$ (DIF) $<=8 *$ LSD THEN RDMS\&RESPONSE $=8$; IF $6 *$ LSD $<$ ABS(DIF) $<=7 *$ LSD THEN RDMS\&RESPONSE $=7$; IF 5 "LSD $<A B S$ (DIF)<=6"LSD THEN RDMS\&RESPONSE $=6$; IF 4 "LSD $<A B S$ (DIF)<=5"LSD THEN RDMS\&RESPONSE $=5$; IF $3 *$ LSD $<A B S$ (DIF) $<=4 *$ LSD THEN RDMS\&RESPONSE $=4$; IF $2^{*}$ LSD $<$ ABS(DIF)<=3*LSD THEN RDMS\&RESPONSE $=3$; IF LSD $<A B S$ (DIF) $<=2 *$ LSD THEN RDMS\&RESPONSE $=2$; IF ABS(DIF)<=LSD THEN RDMS\&RESPONSE $=1$; IF DIF<0 THEN RDMS\&RESPONSE=RDMS\&RESPONSE* $(-1)$;

DROP TYPE_FREQ;

PROC SORT $\bar{D} A \bar{T} A=P R U$ UEAST; BY AJ\&RESPONSE ;

DATA R1;

SET PRUEBAST;

AJ\&RESPONSE=ROUND(AJ\&RESPONSE,.00001) ;

$Y=L A G(A J \& R E S P O N S E)$;

DATA RANGOS;

RANK\&RESPONSE $=1$; 
LOOP: RANK\&RESPONSE=RANK\&RESPONSE;

SET R1; IF Y='.' THEN Y=AJ\&RESPONSE ;

IF AJ\&RESPONSE $=Y$ THEN RANK\&RESPONSE=RANK\&RESPONSE;

ELSE RANK\&RESPONSE=RANK\&RESPONSE+1; OUTPUT;

GOTO LOOP;

DATA IS;

SET RANGOS;

IS\&RESPONSE=RDMS\&RESPONSE+RANK\&RESPONSE;

PROC MEANS DATA=IS NWAY NOPRINT;

VAR IS\&RESPONSE;

OUTPUT OUT=SI MEAN=MIS\&RESPONSE;

DATA IS\&RESPONSE;

SET $S 1$;

LOOP: SET IS;

IF IS\&RESPONSE>MIS\&RESPONSE THEN SELC\&RESPONSE='SI'; ELSE

SELC\&RESPONSE='NO';

IF DIF $>0$ AND PROBT < $<\& A L P H A$ THEN TRAD\&RESPONSE='SUPER' ;

IF DIF $<0$ AND PROBT $<=\& A L P H A$ THEN TRAD\&RESPONSE='INFER' ;

IF PROBT>\&ALPHA THEN TRAD\&RESPONSE='IGUAL' ;

OUTPUT;

GOTO LOOP;

PROC SORT; BY AJ\&RESPONSE;

PROC PRINT;

VAR TRAT \&RESPONSE AJ\&RESPONSE DIF PROBT TRAD\&RESPONSE

RDMS\&RESPONSE RANK\&RESPONSE

IS\&RESPONSE SELC\&RESPONSE;

TITLE 'DECISION DE SELECCION DE NUEVOS GENOTIPO SEGUN EL MEDODO TRADICIONAL';

TITLE2 'Y EL NUEVO MÉTODO DE SUMA DE RANGOS' ;

PROC MEANS DATA=IS\&RESPONSE;

VAR MIS\&RESPONSE LSD;

TITLE 'DECISION DE SELECCION DE NUEVOS GENOTIPO SEGUN EL MEDODO TRADICIONAL';

TITLE2 'Y EL NUEVO METODO DE SUMA DE RANGOS';

TITLE3 'MEDIAS DE LOS INDICES DE SELECCION';

TITLE4 'COMPROBAR QUE "Std Dev" SEA IGUAL A CERO';

PROC FREQ DATA=IS\&RESPONSE;

TABLES TRAD\&RESPONSE*SELC\&RESPONSE;

TITLE 'RELACIÓN ENTRE LAS DOS SELECCIONES';

PROC SORT DATA=IS\&RESPONSE; BY TRAT;

\%MEND DA;

La MACRO DA no es conveniente si se presenta desbalance de los genotipos testigo, lo que es una condición poco deseable para el investigador.

\section{Ejemplo numérico}

Los datos a continuación utilizados provienen de un Diseño Aumentado bajo un Diseño de Bloques Completos al Azar, realizado durante el año de 1999, por el programa de mejoramiento en papa, 
de la Facultad de Agronomía, de la Universidad Nacional de Colombia, Sede Bogotá.

Se utilizaron 6 bloques, 3 variedades testigo (Parda Pastusa, DIACOL Capiro e ICA Única) y se evaluaron 67 genotipos, en unidades experimentales de $20 \mathrm{~m}^{2}$. Las variables evaluadas fueron rendimiento (kg por parcela), vigor (VIG) y precocidad (PREC), estas últimas evaluadas mediante una escala con valores de 1 a 5 . El rendimiento se dividió en tres categorías de acuerdo con el tamaño del tubérculo en, primera (P1), segunda (P2) y tercera (P3). Como regla de decisión se optó por el promedio de los genotipos testigos (opción MEAN, en la MAGRO DA).

\section{Resultados}

Para facilitar la comprensión de las operaciones en la metodología propuesta, se desarrollará el ejercicio sólo para la variable P1. En la Tabla 1, se presenta el cálculo del factor de corrección por cada bloque y en la Tabla 2 el cálculo del IS para esta variable. De igual manera se realizó para las demás variables. En ella es importante resaltar la decisión tomada por el método tradicional, INFERIOR, IGUAL o SUPERIOR al promedio de los testigos (seleccionando los iguales o superiores); y la obtenida por el método propuesto, SI para los superiores al promedio del IS y NO para los inferiores al promedio del IS.

Tabla 1. Datos observados (Yij) para las variedades testigo (variable P1) y obtención del factor de corrección (FC) para cada bloque.

\begin{tabular}{|c|c|c|c|c|c|c|c|}
\hline \multirow{2}{*}{ Genotipo } & \multicolumn{6}{|c|}{ BLOQUE } & \multirow{2}{*}{ Total } \\
\hline & B1 & B2 & B3 & B4 & B5 & B6 & \\
\hline Diacol Capiro & 69.0 & 78.0 & 60.0 & 64.0 & $\overline{71.0}$ & 56.5 & 398.5 \\
\hline Parda Pastusa & 44.5 & 46.5 & 48.0 & 32.5 & 38.0 & 33.5 & 243.0 \\
\hline ICA Unica & 100.5 & 109.5 & 119.0 & 113.5 & 108.5 & 75.0 & 626.0 \\
\hline Total & 214.0 & 234.0 & 227.0 & 210.0 & 217.5 & 165.0 & $1,267.5$ \\
\hline Factor de corrección & 0.92 & 7.58 & 5.25 & -0.42 & 2.08 & -15.41 & \\
\hline
\end{tabular}


Tabla 2. Cálculo del índice de selección (IS) para la variable P1 (Salida SAS MAGRO DA).

\begin{tabular}{|c|c|c|c|c|c|c|c|c|}
\hline Genotipo & Bloque & $\mathrm{P} 1$ & $\mathrm{FC}$ & $\overline{\text { AJP1 }}$ & RankP1 & RDMSP1 & ISP1 & TRADP1 \\
\hline 1 & B3 & 15.00 & 5.25 & 9.75 & 1 & -4.00 & -3 & INFERIOR \\
\hline 2 & B4 & 17.50 & -0.42 & 17.92 & 2 & -3.00 & -1 & INFERIOR \\
\hline 5 & B3 & 27.00 & 5.25 & 21.75 & 3 & -3.00 & 0 & INFERIOR \\
\hline 4 & B4 & 26.50 & -0.42 & 26.92 & 4 & -3.00 & 1 & INFERIOR \\
\hline 12 & $B$ & 39.00 & 7.58 & 31.42 & 5 & -2.00 & 3 & INFERIOR \\
\hline 14 & B2 & 40.00 & 7.58 & 32.42 & 6 & -2.00 & 4 & INFERIOR \\
\hline 9 & B3 & 38.00 & 5.25 & 32.75 & 7 & -2.00 & 5 & INFERIOR \\
\hline 7 & B5 & 35.00 & 2.08 & 32.92 & 8 & -2.00 & 6 & INFERIOR \\
\hline 3 & B6 & 18.00 & -15.42 & 33.42 & 9 & -2.00 & 7 & INFERIOR \\
\hline 16 & B2 & 44.00 & 7.58 & 36.42 & 10 & -2.00 & 8 & INFERIOR \\
\hline 17 & B2 & 44.00 & 7.58 & 36.42 & 10 & -2.00 & 8 & INFERIOR \\
\hline 10 & B4 & 38.00 & -0.42 & 38.42 & 11 & -2.00 & 9 & INFERIOR \\
\hline 18 & B3 & 44.00 & 5.25 & 38.75 & 12 & -2.00 & 10 & INFERIOR \\
\hline 20 & B3 & 44.50 & 5.25 & 39.25 & 13 & -2.00 & 11 & INFERIOR \\
\hline 22 & B2 & 48.00 & 7.58 & 40.42 & 14 & -2.00 & 12 & INFERIOR \\
\hline 19 & B5 & 44.00 & 2.08 & 41.92 & 15 & -2.00 & 13 & INFERIOR \\
\hline 23 & B3 & 48.00 & 5.25 & 42.75 & 16 & -2.00 & 14 & INFERIOR \\
\hline 24 & B3 & 48.00 & 5.25 & 42.75 & 16 & -2.00 & 14 & INFERIOR \\
\hline 25 & B3 & 49.00 & 5.25 & 43.75 & 17 & -2.00 & 15 & INFERIOR \\
\hline 6 & B6 & 30.00 & -15.42 & 45.42 & 18 & -2.00 & 16 & INFERIOR \\
\hline 21 & B5 & 47.50 & 2.08 & 45.42 & 18 & -2.00 & 16 & INFERIOR \\
\hline 26 & B5 & 49.00 & 2.08 & 46.92 & 19 & -2.00 & 17 & INFERIOR \\
\hline 31 & B2 & 55.00 & 7.58 & 47.42 & 20 & -2.00 & 18 & INFERIOR \\
\hline 30 & B3 & 53.50 & 5.25 & 48.25 & 21 & -2.00 & 19 & INFERIOR \\
\hline 8 & B6 & 36.00 & -15.42 & 51.42 & 22 & -1.00 & 21 & IGUAL \\
\hline 38 & B2 & 59.50 & 7.58 & 51.92 & 23 & -1.00 & 22 & IGUAL \\
\hline 29 & B1 & 53.00 & 0.92 & 52.08 & 24 & -1.00 & 23 & IGUAL \\
\hline 28 & B4 & 52.00 & -0.42 & 52.42 & 25 & -1.00 & 24 & IGUAL \\
\hline 11 & B6 & 38.00 & -15.42 & 53.42 & 26 & -1.00 & 25 & IGUAL \\
\hline 13 & B6 & 39.50 & -15.42 & 54.92 & 27 & -1.00 & 26 & IGUAL \\
\hline 33 & B5 & 57.00 & 2.08 & 54.92 & 27 & -1.00 & 26 & IGUAL \\
\hline 34 & B5 & 57.00 & 2.08 & 54.92 & 27 & -1.00 & 26 & IGUAL \\
\hline 40 & B3 & 61.00 & 5.25 & 55.75 & 28 & -1.00 & 27 & IGUAL \\
\hline 15 & B6 & 41.00 & -15.42 & 56.42 & 29 & -1.00 & 28 & IGUAL \\
\hline 32 & B4 & 56.00 & -0.42 & 56.42 & 29 & -1.00 & 28 & IGUAL \\
\hline 36 & B5 & 58.50 & 2.08 & 56.42 & 29 & -1.00 & 28 & IGUAL \\
\hline
\end{tabular}




\begin{tabular}{|l|rrrrrrrl}
\hline Genotipo & Bloque & P1 & FC & AJP1 & RankP1 & RDMSP1 & ISP1 & TRADP1 \\
\hline 35 & B1 & 57.50 & 0.92 & 56.58 & 30 & -1.00 & 29 & IGUAL \\
42 & B3 & 63.00 & 5.25 & 57.75 & 31 & -1.00 & 30 & IGUAL \\
37 & B1 & 59.00 & 0.92 & 58.08 & 32 & -1.00 & 31 & IGUAL \\
39 & B1 & 59.50 & 0.92 & 58.58 & 33 & -1.00 & 32 & IGUAL \\
50 & B2 & 67.00 & 7.58 & 59.42 & 34 & -1.00 & 33 & IGUAL \\
43 & B5 & 63.00 & 2.08 & 60.92 & 35 & -1.00 & 34 & IGUAL \\
46 & B1 & 64.00 & 0.92 & 63.08 & 36 & -1.00 & 35 & IGUAL \\
52 & B3 & 68.50 & 5.25 & 63.25 & 37 & -1.00 & 36 & IGUAL \\
44 & B4 & 63.00 & -0.42 & 63.42 & 38 & -1.00 & 37 & IGUAL \\
55 & B2 & 71.00 & 7.58 & 63.42 & 38 & -1.00 & 37 & IGUAL \\
49 & B5 & 66.50 & 2.08 & 64.42 & 39 & -1.00 & 38 & IGUAL \\
27 & B6 & 49.50 & -15.42 & 64.92 & 40 & -1.00 & 39 & IGUAL \\
47 & B1 & 66.00 & 0.92 & 65.08 & 41 & -1.00 & 40 & IGUAL \\
48 & B1 & 66.00 & 0.92 & 65.08 & 41 & -1.00 & 40 & IGUAL \\
51 & B1 & 68.00 & 0.92 & 67.08 & 42 & -1.00 & 41 & IGUAL \\
53 & B1 & 69.00 & 0.92 & 68.08 & 43 & -1.00 & 42 & IGUAL \\
61 & B2 & 76.50 & 7.58 & 68.92 & 44 & -1.00 & 43 & IGUAL \\
63 & B2 & 77.50 & 7.58 & 69.92 & 45 & -1.00 & 44 & IGUAL \\
56 & B1 & 71.00 & 0.92 & 70.08 & 46 & -1.00 & 45 & IGUAL \\
54 & B4 & 70.00 & -0.42 & 70.42 & 47 & 1.00 & 48 & IGUAL \\
64 & B2 & 79.00 & 7.58 & 71.42 & 48 & 1.00 & 49 & IGUAL \\
57 & B4 & 71.50 & -0.42 & 71.92 & 49 & 1.00 & 50 & IGUAL \\
60 & B5 & 76.00 & 2.08 & 73.92 & 50 & 1.00 & 51 & IGUAL \\
59 & B4 & 74.50 & -0.42 & 74.92 & 51 & 1.00 & 52 & IGUAL \\
62 & B1 & 77.00 & 0.92 & 76.08 & 52 & 1.00 & 53 & IGUAL \\
41 & B6 & 62.00 & -15.42 & 77.42 & 53 & 1.00 & 54 & IGUAL \\
45 & B6 & 63.50 & -1.5 .42 & 78.92 & 54 & 1.00 & 55 & IGUAL \\
65 & B4 & 82.00 & -0.42 & 82.42 & 55 & 1.00 & 56 & IGUAL \\
66 & B4 & 83.00 & -0.42 & 83.42 & 56 & 1.00 & 57 & IGUAL \\
58 & B6 & 74.00 & -15.42 & 89.42 & 57 & 1.00 & 58 & IGUAL \\
67 & B5 & 104.0 & 2.08 & 101.92 & 58 & 2.00 & 60 & SUPERIOR \\
\hline
\end{tabular}

CME $=69.125$; DMS ajustada $=19.82 ;$ Promedio de los testigos $=70.41$; Promedio de ISP1 $=27.98<=$ Genotipos seleccionados

Hay que tener en cuenta que, para las variables P3 y PREC, la regla de decisión para la selección de genotipos se invierte, tanto para el método tradicional como para el propuesto. Así se espera encontrar genotipos inferiores o iguales al promedio de los testigos, y se 
seleccionan los genotipos por debajo del promedio del IS. Esto debido a que, P3 es una producción no comercial y por lo tanto indeseable como característica, y PREC como consecuencia del uso de una escala donde 1 es muy precoz y 5 muy tardío. Además, para el ejercicio se propone el objetivo del mejoramiento la selección de materiales precoces.

Ningún genotipo catalogado como inferior (P1, P2 y VIG) o superior (P3 y PREC), por el método tradicional, fue seleccionado por el nuevo método, en todas las variables (Tabla 3 y 4). De igual forma, ningún genotipo catalogado como superior ( $P 1, P 2$ y VIG) o inferior (P3 y PREC), por el método tradicional, fue descartado por el nuevo método (Tabla 3 y 4). La diferencia está, en cuántos de los genotipos seleccionados por el método tradicional, en este caso de la variable P1 (ISP1), se descartan por el nuevo método. Para nuestro ejemplo fueron 9 genotipos (13.43\%) (Tabla 3). Teniendo en cuenta el resto de las variables evaluadas, (Tabla 3 y 4 ), el porcentaje de genotipos descartables llegó a ser hasta del $46.27 \%$ (ISP3).

Tabla 3. Comparación de la selección por el método tradicional y el método propuesto para las variables P1, P2 y VIG.

\begin{tabular}{|l|lcc|lccc|lcc|}
\hline \multirow{2}{*}{$\begin{array}{c}\text { Método } \\
\text { tradicional }\end{array}$} & \multicolumn{8}{|c|}{ Método propuesto } \\
\cline { 2 - 11 } & \multicolumn{3}{|c|}{ ISP1 } & \multicolumn{3}{c|}{ ISP2 } & \multicolumn{3}{c|}{ ISVIG } \\
\cline { 2 - 11 } & No & Si & Total & No & Si & Total & No & Si & Total \\
\hline Inferior & 24 & 0 & 24 & 25 & 0 & 25 & 20 & 0 & 20 \\
\hline & $35.82^{2}$ & 0.00 & 35.82 & 37.31 & 0.00 & 37.31 & 29.85 & 0.00 & 29.85 \\
\hline Igual & 9 & 33 & 42 & 10 & 25 & 35 & 18 & 26 & 44 \\
\hline & 13.43 & 49.25 & 62.69 & 37.31 & 37.31 & 52.24 & 26.87 & 38.81 & 65.67 \\
\hline Superior & 0 & 1 & 1 & 0 & 7 & 7 & 0 & 3 & 3 \\
\hline & 0.00 & 1.49 & 1.49 & 0.00 & 10.45 & 10.45 & 0.00 & 4.48 & 4.48 \\
\hline Total & 33 & 34 & 67 & 35 & 32 & 67 & 38 & 29 & 67 \\
\hline & 19.25 & 50.75 & 100.0 & 52.24 & 47.76 & 100.0 & 56.72 & 43.28 & 100.0 \\
\hline
\end{tabular}

${ }^{1}$ Frecuencia; ${ }^{2}$ Porcentaje.

Para las variable $\mathrm{P} 1, \mathrm{P} 2$ y VIG, mientras que por el método tradicional la selección de genotipos evaluados osciló entre un $62.69 \%$ (ISP2) y un $70.15 \%$ (ISVIG), por el nuevo método se seleccionó entre un $43.28 \%$ (ISVIG) y un $50.75 \%$ (ISP1) (Tabla 3). Con las variable P3 y PREC, con selección invertida, el método 
tradicional seleccionó respectivamente el $100 \%$ y el $89.55 \%$, de los genotipos evaluados; mientras el método propuesto seleccionó el $53.73 \%$ y el $61.19 \%$ de ellos, respectivamente (Tabla 3 ).

Tabla 4. Comparación de la selección por el método tradicional y el método propuesto para las variables $P 3$ y PREC (variables de selección invertida).

\begin{tabular}{|l|rrr|rrr|}
\hline \multirow{3}{*}{$\begin{array}{l}\text { Método } \\
\text { tradicional }\end{array}$} & \multicolumn{6}{|c|}{ Método propuesto } \\
\cline { 2 - 8 } & \multicolumn{3}{|c}{ ISP3 } & & \multicolumn{3}{c|}{ ISPREC } \\
\cline { 2 - 8 } & No & Si & Total & No & Si & Total \\
\hline Inferior & $0^{1}$ & 0 & 0 & 0 & 41 & 41 \\
\hline & $0.00^{2}$ & 0.00 & 0.00 & 0.00 & 61.19 & 61.19 \\
\hline Igual & 31 & 36 & 67 & 19 & 0 & 19 \\
\hline Superior & 46.27 & 53.73 & 100.00 & 28.36 & 0.00 & 28.36 \\
\hline & 0 & 0 & 0 & 7 & 0 & 7 \\
\hline Total & 0.00 & 0.00 & 0.00 & 10.45 & 0.00 & 10.45 \\
\hline & 31 & 36 & 67 & 26 & 41 & 67 \\
\hline
\end{tabular}

${ }^{1}$ Frecuencia; ${ }^{2}$ Porcentaje

Teniendo en cuenta los anteriores resultados, vemos cómo el método propuesto ayuda a realizar mejor selección de los genotipos, evitando así, que el programa de mejoramiento se llene de material indeseable, que en un futuro lo que implica son mayores costos.

Si consideramos todas las variables evaluadas para adelantar el proceso de selección, consideramos como ejemplo que, el programa de mejoramiento está interesado en comparar dos tipos de selección. La primera utilizando los siguientes coeficientes 15:15:10:16:24, para P1:P2:P3:VIG:PREC, asignando un $50 \%$ de importancia al rendimiento, un $20 \%$ a vigor y un $30 \%$ a precocidad. El segundo, 9:9:6:16 para P1:P2:P3:PREC, asignando un $60 \%$ de importancia a rendimiento y un $40 \%$ a precocidad. Dentro de rendimiento, en ambas selecciones, se le otorgó un $75 \%$ de importancia a $\mathrm{P} 1$ más $\mathrm{P} 2$, y un $25 \%$ a P3, para ellos se obtendrán los índices de selección ISG1 e ISG2, respectivamente (Tabla 5). Como, producción de tubérculos de tercera (P3) y precocidad (PREC), tienen selección invertida, se restan (invierten el signo), para la obtención del ISG. Así por ejemplo ISG1 $=\left(15^{\star} \mid S P 1\right)+\left(15^{*} I S P 2\right)-\left(10^{*} I S P 3\right)+\left(16^{*} I S V I G\right)-\left(24^{*} I S P R E C\right)$. 
Si comparamos la selección realizada por el ISG1 y el ISG2, contra la realizada por el método tradicional para la variable P1 (la que hubiera sido realizada en ausencia de otras posibilidades), encontramos que se están rescatando, respectivamente, el $10.45 \%$ y el $8.96 \%$ de los genotipos (Tabla 5), los cuales hubieran sido descartados por el método tradicional. Además, ningún genotipo seleccionado por este método como superior, fue rechazado por el nuevo método. Nuevamente, la diferencia radica en los genotipos declarados como iguales. El $23.88 \%$ y el $19.40 \%$, del total de genotipos evaluados, que se hubiera seleccionado como iguales al promedio de los genotipos testigos, es descartado por el nuevo método (Tabla 5). En general, mientras que por el método tradicional se seleccionaría el $64.18 \%$, por los análisis propuestos, se seleccionarían el 50.75 y el $53.73 \%$ de los genotipos. 
Tabla 5. Índices de selección para cada variable y para dos tipos de selección multivariada.

\begin{tabular}{|c|c|c|c|c|c|c|c|c|}
\hline Genotipo & ISP1 & ISP2 & ISP3 & ISVIC & & ISPREC & ISG1 & ISG2 \\
\hline 4 & 1 & 3 & $2 \leq$ & 0 & & $2 \leq$ & -8 & -8 \\
\hline 3 & 0 & 9 & 11 & 1 & & $2 \leq$ & -7 & -17 \\
\hline 1 & 10 & 6 & $6 \leq$ & 5 & & 7 & 92 & -4 \\
\hline 11 & 8 & -2 & $1 \leq$ & -2 & & $-2 \leq$ & 96 & 80 \\
\hline 10 & 8 & 13 & 18 & -2 & & $-2 \leq$ & 151 & 113 \\
\hline 19 & 16 & 5 & $4 \leq$ & 5 & & 7 & 187 & 53 \\
\hline 17 & 14 & 10 & $3 \leq$ & 1 & & $2 \leq$ & 298 & 166 \\
\hline 15 & 13 & 18 & $2 \leq$ & 2 & & 7 & 309 & 155 \\
\hline 22 & 18 & 4 & $1 \leq$ & -2 & & $-2 \leq$ & 336 & 224 \\
\hline 1 & -3 & $45 \leq$ & 20 & 14 & $\leq$ & 13 & 342 & 50 \\
\hline 12 & 9 & $30 \leq$ & 13 & 4 & & 7 & 351 & 161 \\
\hline 24 & 21 & 3 & $5 \leq$ & 1 & & $-2 \leq$ & 374 & 218 \\
\hline 28 & 25 & 11 & $8 \leq$ & 10 & $\leq$ & 10 & 380 & 116 \\
\hline 40 & $33 \leq$ & 4 & 9 & 6 & $\leq$ & 7 & 393 & 167 \\
\hline 27 & 24 & 8 & 10 & 0 & & $-2 \leq$ & 428 & 260 \\
\hline 26 & 23 & 7 & 13 & 1 & & $-4 \leq$ & 432 & 256 \\
\hline 2 & -1 & $42 \leq$ & $5 \leq$ & 4 & & 7 & 461 & 227 \\
\hline 16 & 14 & 23 & 20 & 5 & & $-2 \leq$ & 483 & 245 \\
\hline 30 & 26 & 9 & $2 \leq$ & 2 & & $2 \leq$ & 489 & 271 \\
\hline 55 & $48 \leq$ & 9 & 19 & 0 & & 7 & 497 & 287 \\
\hline 33 & $28 \leq$ & 0 & $4 \leq$ & 5 & & $-2 \leq$ & 508 & 260 \\
\hline 52 & $43 \leq$ & -2 & $1 \leq$ & -2 & & $2 \leq$ & 525 & 331 \\
\hline 8 & 6 & $35 \leq$ & 16 & 2 & & $-2 \leq$ & 535 & 305 \\
\hline 3 & 11 & $37 \leq$ & $6 \leq$ & 5 & & 7 & 572 & 284 \\
\hline 21 & 17 & $24 \leq$ & 16 & 6 & $\leq$ & $-2 \leq$ & 599 & 305 \\
\hline 29 & 26 & 21 & 22 & 5 & & $-2 \leq$ & 613 & 323 \\
\hline 44 & $37 \leq$ & 2 & $2 \leq$ & 0 & & $-2 \leq$ & 613 & $371 \leq$ \\
\hline 7 & 5 & $39 \leq$ & 20 & 10 & $\leq$ & $-2 \leq$ & 668 & 308 \\
\hline 53 & $44 \leq$ & -2 & $1 \leq$ & 6 & $\leq$ & $2 \leq$ & 668 & 340 \\
\hline 51 & $42 \leq$ & 7 & 13 & 5 & & $0 \leq$ & 685 & 363 \\
\hline 32 & 27 & $38 \leq$ & 20 & 14 & $\leq$ & 13 & 687 & 257 \\
\hline 39 & $32 \leq$ & 16 & 13 & 1 & & $-4 \leq$ & 702 & $418 \leq$ \\
\hline 45 & $37 \leq$ & 1 & $1 \leq$ & 6 & $\leq$ & $-2 \leq$ & 704 & $368 \leq$ \\
\hline 20 & 16 & $27 \leq$ & $8 \leq$ & 6 & $\leq$ & $-2 \leq$ & $709 \leq$ & $371 \leq$ \\
\hline 9 & 7 & $49 \leq$ & $4 \leq$ & 5 & & 7 & $712 \leq$ & $368 \leq$ \\
\hline 14 & 12 & $44 \leq$ & 12 & 11 & $\leq$ & 7 & $728 \leq$ & 320 \\
\hline
\end{tabular}


Continuación .....

\begin{tabular}{|c|c|c|c|c|c|c|c|c|}
\hline Genotipo & ISP1 & ISP2 & & ISP3 & ISVIG & ISPREC & ISG1 & ISG2 \\
\hline 5 & 3 & 41 & $\leq$ & $1 \leq$ & 2 & $-2 \leq$ & $730 \leq$ & $422 \leq$ \\
\hline 36 & $29 \leq$ & 34 & $\leq$ & 16 & 5 & 4 & $769 \leq$ & $407 \leq$ \\
\hline 41 & $34 \leq$ & 17 & & $0 \leq$ & $6 \leq$ & $2 \leq$ & $813 \leq$ & $427 \leq$ \\
\hline 31 & 26 & 20 & & $2 \leq$ & $6 \leq$ & $-2 \leq$ & $814 \leq$ & $434 \leq$ \\
\hline 18 & 15 & 54 & $\leq$ & 20 & $10 \leq$ & 7 & $827 \leq$ & $389 \leq$ \\
\hline 6 & 4 & 53 & $\leq$ & $1 \leq$ & 2 & $2 \leq$ & $829 \leq$ & $475 \leq$ \\
\hline 23 & 19 & 30 & $\leq$ & 11 & $10 \leq$ & $-2 \leq$ & $833 \leq$ & $407 \leq$ \\
\hline 59 & $52 \leq$ & 9 & & $2 \leq$ & $9 \leq$ & 7 & $871 \leq$ & $425 \leq$ \\
\hline 67 & $60 \leq$ & 9 & & $2 \leq$ & $11 \leq$ & 13 & $879 \leq$ & $401 \leq$ \\
\hline 42 & $35 \leq$ & 29 & $\leq$ & 13 & $10 \leq$ & 4 & $894 \leq$ & $434 \leq$ \\
\hline 49 & $40 \leq$ & 22 & & 21 & 5 & $-4 \leq$ & $896 \leq$ & $496 \leq$ \\
\hline 54 & $45 \leq$ & 19 & & 13 & 5 & $0 \leq$ & $910 \leq$ & $498 \leq$ \\
\hline 58 & $51 \leq$ & 15 & & $0 \leq$ & $6 \leq$ & 7 & $918 \leq$ & $482 \leq$ \\
\hline 35 & $28 \leq$ & 40 & $\leq$ & $5 \leq$ & 0 & $2 \leq$ & $922 \leq$ & $550 \leq$ \\
\hline 51 & $54 \leq$ & 12 & & $8 \leq$ & 5 & $2 \leq$ & $942 \leq$ & $514 \leq$ \\
\hline 46 & $38 \leq$ & 33 & $\leq$ & $5 \leq$ & $6 \leq$ & 7 & $943 \leq$ & $497 \leq$ \\
\hline 47 & $39 \leq$ & 20 & & $4 \leq$ & 5 & $-2 \leq$ & $973 \leq$ & $539 \leq$ \\
\hline 38 & $31 \leq$ & 43 & $\leq$ & 19 & $10 \leq$ & 4 & $984 \leq$ & $488 \leq$ \\
\hline 43 & $36 \leq$ & 50 & $\leq$ & 20 & 5 & 7 & $1002 \leq$ & $542 \leq$ \\
\hline 50 & $41 \leq$ & 26 & $\leq$ & 13 & 5 & $-4 \leq$ & $1051 \leq$ & $589 \leq$ \\
\hline 63 & $56 \leq$ & 9 & & $2 \leq$ & $9 \leq$ & $2 \leq$ & $1051 \leq$ & $541 \leq$ \\
\hline 37 & $30 \leq$ & 52 & $\leq$ & 17 & $10 \leq$ & 7 & $1052 \leq$ & $524 \leq$ \\
\hline 34 & $28 \leq$ & 36 & $\leq$ & $5 \leq$ & $6 \leq$ & $-2 \leq$ & $1054 \leq$ & $578 \leq$ \\
\hline 56 & $49 \leq$ & 28 & $\leq$ & $7 \leq$ & $6 \leq$ & $2 \leq$ & $1133 \leq$ & $619 \leq$ \\
\hline 57 & $50 \leq$ & 37 & $\leq$ & $5 \leq$ & $12 \leq$ & 13 & $1135 \leq$ & $545 \leq$ \\
\hline 54 & $57 \leq$ & 35 & $\leq$ & 10 & $12 \leq$ & 13 & $1160 \leq$ & $560 \leq$ \\
\hline 25 & 22 & 51 & $\leq$ & $1 \leq$ & 2 & $-2 \leq$ & $1165 \leq$ & $683 \leq$ \\
\hline 48 & $40 \leq$ & 46 & $\leq$ & 13 & $14 \leq$ & 9 & $1168 \leq$ & $552 \leq$ \\
\hline 65 & $58 \leq$ & 25 & $\leq$ & 16 & $10 \leq$ & $2 \leq$ & $1197 \leq$ & $619 \leq$ \\
\hline so & $53 \leq$ & 48 & $\leq$ & 13 & 5 & $0 \leq$ & $1465 \leq$ & $831 \leq$ \\
\hline 62 & $55 \leq$ & 37 & $\leq$ & $4 \leq$ & $10 \leq$ & $-2 \leq$ & $1548 \leq$ & $836 \leq$ \\
\hline DMS & 19.82 & 23.4 & & 4.70 & 1.14 & 0.53 & - & - \\
\hline $\begin{array}{l}\text { ajustada(10\%) } \\
\text { Promedio } \\
\text { Testigos }\end{array}$ & 70.41 & 20.5 & & 2.50 & 3.94 & 3.05 & - & - \\
\hline Promedio IS & 27.98 & 11.2 & & 8.95 & 5.35 & 2.62 & 367.40 & 705.07 \\
\hline
\end{tabular}

$\leq$ Genotipos seleccionados. 
Tabla 6. Comparación de la selección por el método tradicional (P1) y los índices de selección multivariados propuestos (ISG1 e ISG2).

\begin{tabular}{|l|rrr|rrr|}
\hline \multirow{2}{*}{ P1 } & \multicolumn{3}{|c|}{ ISG1 } & \multicolumn{3}{c|}{ ISG2 } \\
\cline { 2 - 7 } & No & Si & Total & No & Si & Total \\
\hline Inferior & $17^{1}$ & 7 & 24 & 18 & 6 & 24 \\
& $25 . S 7^{2}$ & 10.45 & 35.82 & 26.87 & 8.96 & 35.82 \\
\hline Igual & 16 & 26 & 42 & 13 & 29 & 42 \\
& 23.88 & 38.81 & 62.69 & 19.40 & 43.28 & 62.69 \\
\hline Superior & 0 & 1 & 1 & 0 & 1 & 1 \\
& 0.00 & 1.49 & 1.49 & 0.00 & 1.49 & 1.49 \\
\hline Total & 33 & 34 & 67 & 31 & 36 & 67 \\
& 49.25 & 50.75 & 100.00 & 46.27 & 53.73 & 100.00 \\
\hline
\end{tabular}

${ }^{1}$ frecuencia; ${ }^{2}$ Porcentaje.

\section{Conclusiones}

El criterio de selección propuesto para los diseños aumentados, mediante los índices de selección, flexibilizan y mejoran las decisiones en los procesos de selección frente al sistema tradicional. La utilización del índice de selección multivariado, traduce los intereses del investigador, en un índice que le ayuda en la toma de decisiones sobre los genotipos promisorios a avanzar para el siguiente ciclo de selección, ganando por lo tanto, una mayor eficiencia para el investigador y para el programa de mejoramiento. La implementación de un programa SAS para el cálculo, de estos índices facilita su utilización, favoreciendo la selección de genotipos, en un tiempo corto, acorde con las necesidades de la dinámica de los programas de mejoramiento.

\section{Referencias Bibliográficas}

1. Federer,. W.T. 1956. Augmented design. Hawaiian Plant Record. 55: $191-208$

2. Federer, W. T. 1961. Augmented design with one-way elimination of heterogeneity. Biometrics 17:447-473.

3. Federer, W. T.; Raghavarao, D. 1975. An augmented design. 
Biometrics 31: 29-35.

4. Glaz B.; Dean J.L. 1988. Statistical error rates and their implications in sugarcane clone triáis. Agronomy Journal 80: 560562.

5. Kang, M.S. 1991. Modified rank-sum method for selecting high yielding, stable crop genotypes. Cereal Research Communications 19: 361-364.

6. Kang, M.S. 1993. Simultaneous selection for yield and stability in crop performance trials: Consequences for growers. Agronomy Journal 85: 754-757.

7. Kang, M.S.; Magari, R. 1996. New development in selecting for phenotypic stability in crop'breeding. p. 1-14 en Genotype-byEnvironmet Interaction. Kang M.S. y Gauch H.G. eds. CRC press, New York.

8. Kang, M.S.; Pham, H.N. 1991. Simultaneous selection for high yielding and stable crop genotypes. Agronomy Journal 83: 161165.

9. Pérez O.; Santamaría, S. 1990. Eficiencia en diseño aumentado en pruebas preliminares de rendimiento. Tesis de Ingeniero Agrónomo. Universidad Nacional de Colombia. Facultad de Agronomía, Santafé de Bogotá.

10. Puri M.L.; Sen P.K. 1971. Nonparametric methods in multivariate analysis. John Wiley \& Sons. New York. E.U.A.

11. SAS Institute. 1996. SAS/STAT software release 6.11. SAS. Inst., Cary, N.C. E.U.A. 